\title{
Boundedness of verbal and adjectival predicates in Mandarin
}

\author{
Charles Lam* \\ LSA Annual Meeting, Portland, January 8-11, 2015
}

1. Goal. This paper compares verbal (VP) and adjectival predication (AP) in Mandarin and extends the boundedness account of $b a$-construction to transitive comparatives (Erlewine, 2007).

2. Problem. Mandarin VP and AP show alternations that have seemingly the same semantics. However, they differ in their selection of predicates. The ba-construction (1a) requires markers of completion, degree achievement, or perfective marker le. Transitive comparatives (Grano \& Kennedy, 2012; Erlewine, 2007) observe the same boundedness constraint. Example (2b) requires a measure phrase and is unacceptable without delimitation by the measure phrase 'a little' or other dimension-appropriate measure phrases, as in ' $2 \mathrm{~cm}$ taller' or ' 5 pounds heavier'.
(1) a. ta ba shu kan *(wan le)
3 sg BA book read finish Perf
'S/he finished reading the book.' ( $b a$ requires either 'finish' or Perf)
b. ta kan (wan) (le) shu
3 sg read finish Perf book
'S/he finished reading the book.' (optional wan and le in SVO)
(2) a. zhuozi gao yizi *(yi cun)
table tall chair one inch
'The table is (an inch) taller than the chair.' (obligatory measure phrase)
b. zhuozi bi gao yizi (yi cun)
table BI tall chair one inch
'The table is (an inch) taller than the chair.' (optional measure phrase)

In addition, existing accounts for the ba-construction (Bender 2000, Huang, Li \& Li 2009, among others) largely focus on the syntactic behaviors of $b a$, but do not take the semantics of the predicates into consideration. The unacceptability of (3) cannot be explained by the syntax alone. Since the predicate xiang 'think' is atelic and the object is not affected, Lipenkova (2011) generalizes that the $b a$-construction must include a scalar predicate and a difference value, which explains why the predicate in (3) can occur in ba-construction and why tai 'too' must be present.

$$
\begin{array}{llllll}
\text { ta } & \text { ba } & \text { zhe-jian-shi xiang } & \text { de } & * \text { (tai) } & \text { beigwan } \\
3 \mathrm{sg} & \mathrm{BA} & \text { this.matter think } & \mathrm{DE} & \text { too } & \text { pessimistic }
\end{array}
$$

'S/he thinks too pessimistic of this matter.' (Lipenkova, 2011)

(3) is unacceptable without 'too' or other degree markers like hen 'very', which indicates assertion of degree. This study builds upon Liu (1997) and Lipenkova (2011) and argues that ba selects only bounded predicates, which can be marked lexically by a secondary predicate like wan 'finish', degree modification containing $d e-\mathrm{XP}$, or the perfective aspect marker $l e$.

3. Proposal. The goal of this study is to find the constraints of the two alternations in verbal and adjectival predicates. This study extends the boundedness constraint of $b a$ and argues that boundedness governs the behavior of both alternations in their predicate selection.

\footnotetext{
*Author: Charles Lam, Purdue University (charleslam@purdue.edu).
} 
Boundedness can be expressed in several ways. First, verbal predicates in Mandarin often consist of more than one verb and form productive VV-compounds, such as chi wan 'eat-finish' in (1a). Second, the verbal predicates often co-occur with perfective marker $l e$, which indicates completion of events. Third, the object of predicates are typically specific or quantized (see Liu (1997) for a generalized notion of NP specificity in relation to $b a$ ). For comparatives, the boundedness can be manifested by a measure phrase, which denotes difference between two degrees. This study posits a common structure for all four variants in VP and AP. The functional head $\mathrm{F}$ selects only bounded predicates as its complement. This rules out the unbounded predicates in the $b a$-construction and transitive comparatives, such as (1a) and (2a).

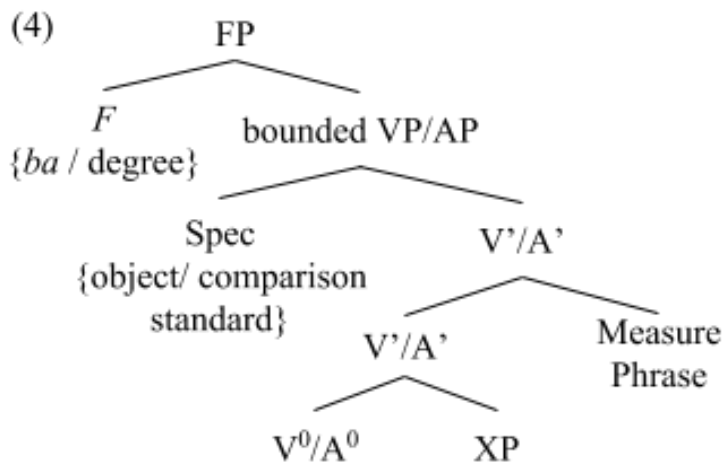

This study hypothesizes that the boundedness constraint is active for VP and AP. The functional head $\mathrm{F}$ selects only bounded predicates (4). When $\mathrm{F}$ is realized by $b a$, it selects a bounded verbal predicate as its complement, which can be lexically bounded (e.g. achievement verbs like sha-si 'kill') or bounded by degree modification (e.g. 'too pessimistic' as in (1)). When F is realized by the comparative affix, it selects bounded adjectival predicates and is spelled out via head movement from $\mathrm{A}^{0}$ to $\mathrm{F}$. The hypothesis is tested by distribution of A-not-A questions, aspect marking and entailment patterns, which support the hypothesized structure (4).

To test whether the complement predicates of $\mathrm{F}$ are truly bounded, I borrow the almost-test (Amaral, 2006; Penka, 2006), which is often used for telicity or scalar properties. One can see that $b a$ and transitive comparatives bear the same ambiguity pattern, i.e. they have both $\mathrm{C}$ (ounterfactual)-interpretation and the $\mathrm{S}$ (calar)-interpretation.

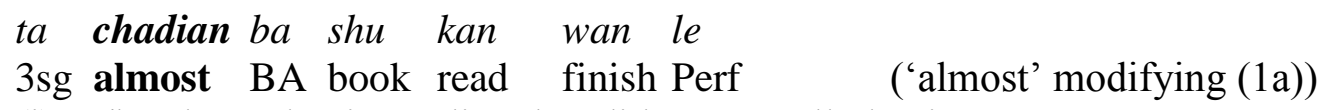

(i) 'S/he almost begin reading, but did not actually begin.' $\quad \rightarrow$ C-interpretation

(ii) 'S/he started reading and almost got to the end.' $\rightarrow$ S-interpretation

$$
\begin{aligned}
& \text { zhuozi chadian gao yizi yi cun } \\
& \text { table almost tall chair one inch ('almost' modifying (2a)) } \\
& \text { (i) 'The table is not taller than the chair, though you thought so.' } \rightarrow \text { C-interpretation } \\
& \text { (ii) 'The table is taller than the chair, but not by as much as an inch' } \rightarrow \text { S-interpretation }
\end{aligned}
$$

The semantic test shows that the predicates are indeed scalar and denotes an endpoint or a degree. The C-interpretation is possible for most predicates. Crucially, the S-interpretation indicates that there is a degree along the scale that has not been reached, and that is the boundary of the predicate. 
4. Remaining issue. The proposal does not fully explain why some adjectives are not compatible with transitive comparatives. Examples in (7) consist of adjectives that denote closed or upperbound scales (Kennedy \& McNally, 2005). (7a) is unacceptable, though their counterparts in bicomparative are acceptable.

a. ??beizi $\{$ man / gan $\}$ pingzi yi-dian
cup full dry bottle a.little
Intended: 'The cup is fuller/drier than the bottle.'
b. beizi bi pingzi $\{$ man / gan $\quad$ yi-dian
cup BI bottle full dry a.little
'The cup is fuller/drier than the bottle.'

Since (7b) is felicitous, it seems that (7a) is unacceptable for syntactic reasons. Specifically, this suggests that head movement from $\mathrm{A}^{0}$ to $\mathrm{F}$ is illicit. One possibility is that closed or upper-bound scale adjectives are phrases, rather than heads. Consequently, the phrase representing the inherently-bounded adjectives cannot move to a head position.

5. Implications. By showing the parallels between VP and AP, this study shows evidence that semantic properties, such as boundedness, can play an active role in syntactic selection. This would contribute to a principled account for how semantics maps with syntax. This homomorphic account also unifies the verbal and adjectival domains in predication. The structure subsumes the syntax and semantics of predication in both domains and is important for a generalized theory. This unified account to verbal and adjectival predication provides a simpler theory that accounts for a larger set of language data, as well as a tight mapping between syntax and semantics in both verbal and adjectival domains, without resorting to category-specific notions like affectedness or telicity. 6. Future Research. An experimental studies is in progress (Lam, in progress 2015) to test the hypothesis regarding acceptability of certain combinations (e.g. ba sentences are predicted to be incompatible with unbounded predicates) and comprehension of predicate boundedness. In addition, the interaction between transitive comparatives and scalar structure will reveal the composition of Mandarin adjectives.

\section{References:}

Amaral, Patricia. (2006). On the semantics of almost. In Annual Meeting of the Linguistics Society of America, University of New Mexico.

Bender, Emily. (2000). The syntax of Mandarin Bă: Reconsidering the verbal analysis. Journal of East Asian Linguistics, 9(2), 105-145.

Erlewine, Michael. (2007). A new syntax-semantics for the Mandarin bi comparative. University of Chicago MA thesis.

Grano, Thomas, \& Christopher Kennedy. (2012). Mandarin transitive comparatives and the grammar of measurement. Journal of East Asian Linguistics, 21(3), 219-266.

Huang, C. T. James, Y. H. Audrey Li,, \& Yafei Li. (2009). The syntax of Chinese. Cambridge: Cambridge University Press.

Lam, Charles. (in progress, 2015). Homomorphic approach to VP and AP alternations. Doctoral dissertation. Purdue University.

Lipenkova, Janna. (2011). Reanalysis of obligatory modifiers as complements in the Chinese ba-construction. In Proceedings of the 18th International Head-driven Phrase Structure Grammar Conference, Stanford: CSLI Publications.

Liu, Feng-Hsi. (1997). An aspectual analysis of ba. Journal of East Asian Linguistics, 6(1), 51-99.

Penka, Doris. (2006). Almost there : the meaning of almost Publ. in: Proceedings of the Sinn und Bedeutung 10:10th annual meeting of the Gesellschaft für Semantik, October 13-15, 2005 / Christian Ebert \& Cornelia Endriss (eds.). Berlin : ZAS, 2006. - S. 275-286. - (ZAS Papers in Linguistics ; 44)

Xiang, Ming. (2005). Some Topics in Comparative Constructions. Ph.D. thesis, Michigan State University. 